\title{
ZnO nanoparticles: An efficient reagent, simple and One-Pot Procedure for Synthesis of Highly Functionalized Dihydropyridine Derivatives
}

\author{
MARZIYEH KHAZAEI ${ }^{1}$, MOHAMMAD ANARY-ABBASINEJAD ${ }^{2}$, ALIREZA \\ HASSANABADI $^{3 *}$, and BAHAREH SADEGHI ${ }^{1}$ \\ ${ }^{1}$ Department of Chemistry, Islamic Azad University, Yazd Branch, P.O. Box 89195-155, \\ Yazd, Iran \\ ${ }^{2}$ Department of Chemistry, Rafsanjan Vali-e-Asr University, Rafsanjan, P.O. Box 77176, \\ Iran \\ ${ }^{3}$ Department of Chemistry, Islamic Azad University, Zahedan Branch, P.O. Box 98135-978, \\ Zahedan, Iran
}

ar_hasanabadi@yahoo.com

Received 20 May 2011; Accepted 15 July 2011

\begin{abstract}
A new and efficient one-pot synthesis of dihydropyridones derivatives by four-component reaction between cyanoacetamide, aryl aldehydes, and ethyl acetoacetate with ammonium acetate using nano $\mathrm{ZnO}$ is described. The reaction was performed in ethanol under reflux conditions and afforded good yields of products.
\end{abstract}

Keywords: Dihydropyridones derivatives, Ammonium acetate, Cyanoacetamide, Aryl aldehydes, Multi-component reaction, $\mathrm{ZnO}$ nanoparticles.

\section{Introduction}

Substituted dihydropyridones derivatives are important intermediates in the pharmaceutical, dye and photo industries. ${ }^{1}$ pyridones are of interest because of the occurrence of their saturated and partially saturated derivatives in biologically active compounds and natural products such as NAD nucleotides, pyridoxol (vitamin $\mathrm{B}_{6}$ ), and pyridine alkaloids. ${ }^{2}$ Due to their $\pi$-stacking ability, some pyridines are used in supramolecular chemistry. ${ }^{3}$ Some examples are used as pharmaceuticals (as antimalarial, vasodilator, anesthetic, anticonvulsant, and antiepileptic), dyes, additives (as antioxidant), agrochemicals (as fungicidal, pesticidal, and herbicidal), veterinary (as anthelmintic, antibacterial, and antiparasitic), and also in qualitative and quantitative analysis ${ }^{4-7}$. So far, the most common synthetic methods for the preparation of pyridine ring systems involve: transformation of another ring, and cyclizations classified on the basis of the number of ring atoms in each of the components being cyclized: from six ring atoms by $N-C_{\alpha}, C_{\alpha}-C_{\beta}$, or $C_{\beta}-C_{\gamma}$ bond 
formation; by formation of two bonds, from [5+1], [4+2], or [3+3] atom fragments; by formation of three bonds, from $[4+1+1],[3+2+1]$, or $[2+2+2]$ atom fragments; and by formation of four bonds, from $[3+1+1+1]$ or $[2+2+1+1]$ atom fragments. ${ }^{8,9}$ Synthesis of 4-aryl-3-cyano2,5-dihydro pyridin-2-one derivatives under solvent-Free conditions we reported ${ }^{10}$.

Nanomaterials have attracted considerable interest in the last decade because of their unique properties in physics and chemistry as well as their potential industrial applications. Among these materials, $\mathrm{ZnO}$ is an important semi-conducting material, having a wide range of properties. The size and morphology of $\mathrm{ZnO}$ nanoparticles have great influences on their performances. Recently, efforts have been made for the synthesis of $\mathrm{ZnO}$ particles with controlled morphologies. Wurtzite-type $\mathrm{ZnO}$ crystals are used for a wide variety of industrial applications, such as semiconductors ${ }^{11}$ catalysts $^{12}$, room-temperature UV lasers ${ }^{13}$, solar cells ${ }^{14}$ and sensors ${ }^{15}$.

\section{Experimental}

Melting points were determined with an Electrothermal 9100 apparatus. Elemental analyses were performed using a Costech ECS 4010 CHNS-O analyzer at analytical laboratory of Islamic Azad University Yazd branch. Mass spectra were recorded on a FINNIGAN-MAT 8430 mass spectrometer operating at an ionization potential of $70 \mathrm{eV}$. IR spectra were recorded on a Shimadzu IR-470 spectrometer. ${ }^{1} \mathrm{H}$ and ${ }^{13} \mathrm{C}$ NMR spectra were recorded on BRUKER DRX-500 AVANCE spectrometer at 500.1 and $125.8 \mathrm{MHz}$, respectively. ${ }^{1} \mathrm{H}$ and ${ }^{13} \mathrm{C}$ NMR spectra were obtained on solution in $\mathrm{CDCl}_{3}$ using TMS as internal standard. Column chromatography was performed with Merck silica gel 60, 230-400 mesh. The chemicals used in this work were purchased from Fluka (Buchs, Switzerland) and were used without further purification.

\section{General procedure}

In a typical experiment, a mixture of $\mathrm{ZnO}$ nanoparticles (10 mol\%), aryl aldehydes $\mathbf{1}$ (1 $\mathrm{mmol})$, and cyanoacetamide $2(1 \mathrm{mmol})$ in ethanol $(20 \mathrm{~mL})$ was stirred at room temperature for $3 \mathrm{~h}$ and was added to it a solution of, ethyl acetoacetate $(1 \mathrm{mmol}) \mathbf{3}$ and $\mathrm{NH}_{4} \mathrm{OAc}(1 \mathrm{mmol})$ and was refluxed for $8 \mathrm{~h}$. After completion of the reaction, as indicated by TLC, the product was extracted with ethyl acetate $(10 \mathrm{~mL})$. The combined organic extracts were concentrated in vacuum and the resulting product was purified by column chromatography on silica gel with ethyl acetate and n-hexane (1:1) as eluent to afford the pure product.

\section{Ethyl 5-cyano-2-methyl-6-oxo-4-phenyl-1,4,5,6-tetrahydropyridine 3-carboxylate (1A)}

White powder, (59\%), m.p. $143-145^{\circ} \mathrm{C}$, IR $(\mathrm{KBr})\left(v_{\max } / \mathrm{cm}^{-1}\right): 3411(\mathrm{NH}), 2234(\mathrm{CN}), 1709$ $(\mathrm{C}=\mathrm{O})$. MS, m/z (\%): $284\left(\mathrm{M}^{+}\right.$, 5). Anal. Calcd for $\mathrm{C}_{16} \mathrm{H}_{16} \mathrm{~N}_{2} \mathrm{O}_{3}: \mathrm{C}, 67.59 ; \mathrm{H}, 5.67 ; \mathrm{N}, 9.85$ \%. Found: C, 67.43; H, 5.72; N, 9.75\%. ${ }^{1} \mathrm{H}$ NMR $\left(500.1 \mathrm{MH}_{\mathrm{Z}}, \mathrm{CDCl}_{3}\right): \delta=0.80\left(3 \mathrm{H}, \mathrm{t},{ }^{3} \mathrm{~J}_{\mathrm{HH}}\right.$ $\left.=7 \mathrm{H}_{\mathrm{Z}}, \mathrm{CH}_{3}\right), 2.38\left(3 \mathrm{H}, \mathrm{s}, \mathrm{CH}_{3}\right), 4.09\left(2 \mathrm{H}, \mathrm{q},{ }^{3} J_{\mathrm{HH}}=7 \mathrm{H}_{\mathrm{Z}}, \mathrm{CH}_{2}\right), 4.16\left(\mathrm{H}, \mathrm{d},{ }^{3} J_{\mathrm{HH}}=7.1 \mathrm{H}_{\mathrm{Z}}\right.$, $\mathrm{CH}), 4.47\left(\mathrm{H}, \mathrm{d},{ }^{3} \mathrm{~J}_{\mathrm{HH}}=7.1 \mathrm{H}_{\mathrm{Z}}, \mathrm{CH}\right), 7.26-7.61(5 \mathrm{H}, \mathrm{m}$, aromatic $), 13.38(1 \mathrm{H}$, broad s, NH). ${ }^{13} \mathrm{C}$ NMR $\left(125.7 \mathrm{MH}_{\mathrm{Z}}, \mathrm{CDCl}_{3}\right): \delta=14.50$ and $19.27\left(2 \mathrm{CH}_{3}\right), 41.66$ and $41.97(2 \mathrm{CH}), 61.23$ $\left(\mathrm{CH}_{2}\right), 108.12(\mathrm{CN}), 114.62$ and $146.48(\mathrm{C}=\mathrm{C}), 128.25,128.95,129.45$, 136.47 (aromatic), 163.71 and $165.91(2 \mathrm{C}=\mathrm{O})$.

Ethyl 5-cyano-2-methyl-6-oxo-4-(4-chlorophenyl)-1,4,5,6-tetrahydropyridine 3carboxylate (2A)

White powder, $(58 \%)$, m.p. $162-164^{\circ} \mathrm{C}$, IR $(\mathrm{KBr})\left(v_{\max } / \mathrm{cm}^{-1}\right): 3403(\mathrm{NH}), 2273(\mathrm{CN}), 1706$ $(\mathrm{C}=\mathrm{O}) . \mathrm{MS}, \mathrm{m} / \mathrm{z}(\%): 318\left(\mathrm{M}^{+}, 10\right)$. Anal. Calcd for $\mathrm{C}_{16} \mathrm{H}_{15} \mathrm{ClN}_{2} \mathrm{O}_{3}: \mathrm{C}, 60.29 ; \mathrm{H}, 4.74 ; \mathrm{N}$, 
8.79\%. Found: C, 60.20; H, 4.87; N, 8.63\%. ${ }^{1} \mathrm{H}$ NMR (500.1 $\left.\mathrm{MH}_{\mathrm{Z}}, \mathrm{CDCl}_{3}\right): \delta=1.21(3 \mathrm{H}, \mathrm{t}$, $\left.{ }^{3} J_{\mathrm{HH}}=7 \mathrm{H}_{\mathrm{Z}}, \mathrm{CH}_{3}\right), 2.49\left(3 \mathrm{H}, \mathrm{s}, \mathrm{CH}_{3}\right), 4.13\left(2 \mathrm{H}, \mathrm{q},{ }^{3} \mathrm{~J}_{\mathrm{HH}}=7 \mathrm{H}_{\mathrm{Z}}, \mathrm{CH}_{2}\right), 3.66\left(\mathrm{H}, \mathrm{d},{ }^{3} J_{\mathrm{HH}}=\right.$ $\left.7.1 \mathrm{H}_{\mathrm{Z}}, \mathrm{CH}\right), 4.48\left(\mathrm{H}, \mathrm{d},{ }^{3} J_{\mathrm{HH}}=7.1 \mathrm{H}_{\mathrm{Z}}, \mathrm{CH}\right), 7.10-7.38(4 \mathrm{H}, \mathrm{m}$, aromatic $), 12.74(1 \mathrm{H}$, broad $\mathrm{s}, \mathrm{NH}) .{ }^{13} \mathrm{C} \mathrm{NMR}\left(125.7 \mathrm{MH}_{\mathrm{Z}}, \mathrm{CDCl}_{3}\right): \delta=14.30$ and $18.51\left(2 \mathrm{CH}_{3}\right), 40,99$ and $41.83(2 \mathrm{CH})$, $60.31\left(\mathrm{CH}_{2}\right), 104.62(\mathrm{CN}), 116.05$ and $148.30(\mathrm{C}=\mathrm{C}), 129.17,130.04,132.99,137.50$ (aromatic), 163.28 and $166.10(2 \mathrm{C}=\mathrm{O})$.

Ethyl 5-cyano-2-methyl-6-oxo-4-(4-bromophenyl)-1,4,5,6-tetrahydropyridine 3-carboxylate (3A)

White powder, $(61 \%)$, m.p. $175-177^{\circ} \mathrm{C}$, IR $(\mathrm{KBr})\left(v_{\max } / \mathrm{cm}^{-1}\right): 3414(\mathrm{NH}), 2210(\mathrm{CN}), 1697$ $(\mathrm{C}=\mathrm{O})$. MS, $\mathrm{m} / \mathrm{z}(\%): 363\left(\mathrm{M}^{+}, 8\right)$. Anal. Calcd for $\mathrm{C}_{16} \mathrm{H}_{15} \mathrm{BrN}_{2} \mathrm{O}_{3}: \mathrm{C}, 52.91 ; \mathrm{H}, 4.16 ; \mathrm{N}$, 7.71\%. Found: $\mathrm{C}, 52.60 ; \mathrm{H}, 4.25 ; \mathrm{N}, 7.61 \%$. ${ }^{1} \mathrm{H}$ NMR $\left(500.1 \mathrm{MH}_{\mathrm{Z}}, \mathrm{CDCl}_{3}\right): \delta=1.22(3 \mathrm{H}, \mathrm{t}$, $\left.{ }^{3} J_{\mathrm{HH}}=7 \mathrm{H}_{\mathrm{Z}}, \mathrm{CH}_{3}\right), 2.46\left(3 \mathrm{H}, \mathrm{s}, \mathrm{CH}_{3}\right), 4.11\left(2 \mathrm{H}, \mathrm{q},{ }^{3} \mathrm{~J}_{\mathrm{HH}}=7 \mathrm{H}_{\mathrm{Z}}, \mathrm{CH}_{2}\right), 3.62\left(\mathrm{H}, \mathrm{d},{ }^{3} J_{\mathrm{HH}}=\right.$ $\left.7.1 \mathrm{H}_{\mathrm{Z}}, \mathrm{CH}\right), 4.47\left(\mathrm{H}, \mathrm{d},{ }^{3} J_{\mathrm{HH}}=7.1 \mathrm{H}_{\mathrm{Z}}, \mathrm{CH}\right), 7.12-7.49(4 \mathrm{H}, \mathrm{m}$, aromatic $), 7.84(1 \mathrm{H}$, broad s, $\mathrm{NH}) .{ }^{13} \mathrm{C}$ NMR $\left(125.7 \mathrm{MH}_{\mathrm{Z}}, \mathrm{CDCl}_{3}\right): \delta=14.12$ and $19.03\left(2 \mathrm{CH}_{3}\right), 40,99$ and $41.07(2 \mathrm{CH})$, $60.97\left(\mathrm{CH}_{2}\right), 107.38(\mathrm{CN}), 113.85$ and $146.05(\mathrm{C}=\mathrm{C}), 122.72,129.35,132.26,134.93$ (aromatic), 162.79 and $165.18(2 \mathrm{C}=\mathrm{O})$.

Ethyl 5-cyano-2-methyl-6-oxo-4-(3-methoxyphenyl)-1,4,5,6-tetrahydropyridine 3-carboxylate (4A)

White powder, (57\%), m.p. $134-136^{\circ} \mathrm{C}$, IR $(\mathrm{KBr})\left(v_{\max } / \mathrm{cm}^{-1}\right): 3415(\mathrm{NH}), 2220(\mathrm{CN}), 1705$ $(\mathrm{C}=\mathrm{O})$. MS, m/z (\%): $314\left(\mathrm{M}^{+}\right.$, 12). Anal. Calcd for $\mathrm{C}_{17} \mathrm{H}_{18} \mathrm{~N}_{2} \mathrm{O}_{4}: \mathrm{C}, 64.96 ; \mathrm{H}, 5.77 ; \mathrm{N}$, 8.91\%. Found: C, 64.88; H, 5.70; N, $8.95 \%$. ${ }^{1} \mathrm{H}$ NMR $\left(500.1 \mathrm{MH}_{\mathrm{Z}}, \mathrm{CDCl}_{3}\right): \delta=1.24(3 \mathrm{H}, \mathrm{t}$, $\left.{ }^{3} J_{\mathrm{HH}}=7 \mathrm{H}_{\mathrm{Z}}, \mathrm{CH}_{3}\right), 2.44\left(3 \mathrm{H}, \mathrm{s}, \mathrm{CH}_{3}\right), 3.80\left(3 \mathrm{H}, \mathrm{s}, \mathrm{OCH}_{3}\right), 4.16\left(2 \mathrm{H}, \mathrm{q},{ }^{3} J_{\mathrm{HH}}=7 \mathrm{H}_{\mathrm{Z}}, \mathrm{CH}_{2}\right)$, $4.10\left(\mathrm{H}, \mathrm{d},{ }^{3} \mathrm{~J}_{\mathrm{HH}}=7.1 \mathrm{H}_{\mathrm{Z}}, \mathrm{CH}\right), 4.48\left(\mathrm{H}, \mathrm{d},{ }^{3} \mathrm{~J}_{\mathrm{HH}}=7.1 \mathrm{H}_{\mathrm{Z}}, \mathrm{CH}\right), 6.79-7.29(4 \mathrm{H}, \mathrm{m}$, aromatic), $7.82\left(1 \mathrm{H}\right.$, broad s, NH). ${ }^{13} \mathrm{C}$ NMR $\left(125.7 \mathrm{MH}_{\mathrm{Z}}, \mathrm{CDCl}_{3}\right): \delta=14.11$ and $18.87\left(2 \mathrm{CH}_{3}\right), 39.82$ and $42.91(2 \mathrm{CH}), 55.23\left(\mathrm{OCH}_{3}\right), 60.82\left(\mathrm{CH}_{2}\right), 107.61(\mathrm{CN}), 113.39$ and $146.02(\mathrm{C}=\mathrm{C})$, $113.08,114.13,120.03,130.07,137.51159 .87$ (aromatic), 163.20 and $165.02(2 \mathrm{C}=\mathrm{O})$.

Ethyl 5-cyano-2-methyl-6-oxo-4-(2-nitrophenyl)-1,4,5,6-tetrahydropyridine 3-carboxylate (5A)

White powder, $(54 \%)$, m.p. $166-168{ }^{\circ} \mathrm{C}$, IR $(\mathrm{KBr})\left(v_{\max } / \mathrm{cm}^{-1}\right): 3400(\mathrm{NH}), 2250(\mathrm{CN}), 1704$ $(\mathrm{C}=\mathrm{O}), 1346$ and $1523\left(\mathrm{NO}_{2}\right) .{ }^{1} \mathrm{H}$ NMR $\left(500.1 \mathrm{MH}_{\mathrm{Z}}, \mathrm{CDCl}_{3}\right): \delta=1.10\left(3 \mathrm{H}, \mathrm{t},{ }^{3} J_{\mathrm{HH}}=7 \mathrm{H}_{\mathrm{Z}}\right.$, $\left.\mathrm{CH}_{3}\right), 2.57\left(3 \mathrm{H}, \mathrm{s}, \mathrm{CH}_{3}\right), 4.04\left(2 \mathrm{H}, \mathrm{q},{ }^{3} \mathrm{~J}_{\mathrm{HH}}=7 \mathrm{H}_{\mathrm{Z}}, \mathrm{CH}_{2}\right), 3.97\left(\mathrm{H}, \mathrm{d},{ }^{3} J_{\mathrm{HH}}=7.1 \mathrm{H}_{\mathrm{Z}}, \mathrm{CH}\right)$, $4.29\left(\mathrm{H}, \mathrm{d},{ }^{3} J_{\mathrm{HH}}=7.1 \mathrm{H}_{\mathrm{Z}}, \mathrm{CH}\right), 7.20-7.98(4 \mathrm{H}, \mathrm{m}$, aromatic $), 8.03\left(1 \mathrm{H}\right.$, broad s, NH). ${ }^{13} \mathrm{C}$ NMR (125.7 $\left.\mathrm{MH}_{\mathrm{Z}}, \mathrm{CDCl}_{3}\right): \delta=13.75$ and $18.91\left(2 \mathrm{CH}_{3}\right), 39.93$ and $41.95(2 \mathrm{CH}), 60.97$ $\left(\mathrm{CH}_{2}\right), 106.66(\mathrm{CN}), 114.94$ and $147.46(\mathrm{C}=\mathrm{C}), 125.58,128.30,129.48,132.90,134.01$, 148.89 (aromatic), 163.08 and $165.22(2 \mathrm{C}=\mathrm{O})$.

Ethyl 5-cyano-2-methyl-6-oxo-4-(3-nitrophenyl)-1,4,5,6-tetrahydropyridine 3-carboxylate (6A)

White powder, (68 \%), m.p. $190-192{ }^{\circ} \mathrm{C}$, IR $(\mathrm{KBr})\left(v_{\max } / \mathrm{cm}^{-1}\right): 3410(\mathrm{NH}), 2230(\mathrm{CN}), 1707$ $(\mathrm{C}=\mathrm{O}), 1319$ and $1525\left(\mathrm{NO}_{2}\right){ }_{1}^{1} \mathrm{H}$ NMR $\left(500.1 \mathrm{MH}_{\mathrm{Z}}, \mathrm{CDCl}_{3}\right): \delta=1.09\left(3 \mathrm{H}, \mathrm{t},{ }^{3} J_{\mathrm{HH}}=7 \mathrm{H}_{\mathrm{Z}}\right.$, $\left.\mathrm{CH}_{3}\right), 2.35\left(3 \mathrm{H}, \mathrm{s}, \mathrm{CH}_{3}\right), 4.01\left(2 \mathrm{H}, \mathrm{q},{ }^{3} \mathrm{~J}_{\mathrm{HH}}=7 \mathrm{H}_{\mathrm{Z}}, \mathrm{CH}_{2}\right), 4.31\left(\mathrm{H}, \mathrm{d},{ }^{3} J_{\mathrm{HH}}=7.1 \mathrm{H}_{\mathrm{Z}}, \mathrm{CH}\right)$, $4.47\left(\mathrm{H}, \mathrm{d},{ }^{3} \mathrm{~J}_{\mathrm{HH}}=7.1 \mathrm{H}_{\mathrm{Z}}, \mathrm{CH}\right), 7.46-8.09(4 \mathrm{H}, \mathrm{m}$, aromatic $), 10.54(1 \mathrm{H}$, broad s, NH$) .{ }^{13} \mathrm{C}$ NMR (125.7 $\left.\mathrm{MH}_{\mathrm{Z}}, \mathrm{CDCl}_{3}\right): \delta=14.04$ and $18.87\left(2 \mathrm{CH}_{3}\right), 44.81$ and $45.94(2 \mathrm{CH}), 65.28$ $\left(\mathrm{CH}_{2}\right), 110.05(\mathrm{CN}), 112.13$ and $153.14(\mathrm{C}=\mathrm{C}), 120.25,127.97,134.80,139.28,144.37$, 153.49 (aromatic), 163.97 and $166.92(2 \mathrm{C}=\mathrm{O})$.

Ethyl 5-cyano-2-methyl-6-oxo-4-(4-nitrophenyl)-1,4,5,6-tetrahydropyridine 3-carboxylate ( $7 A$ ) 
White powder, $(63 \%)$, m.p. $183-185{ }^{\circ} \mathrm{C}$, IR $(\mathrm{KBr})\left(v_{\max } / \mathrm{cm}^{-1}\right): 3407(\mathrm{NH}), 2255(\mathrm{CN}), 1715$ $(\mathrm{C}=\mathrm{O}), 1345$ and $1515\left(\mathrm{NO}_{2}\right) . \mathrm{MS}, \mathrm{m} / \mathrm{z}(\%): 329\left(\mathrm{M}^{+}, 10\right)$. Anal. Calcd for $\mathrm{C}_{16} \mathrm{H}_{13} \mathrm{~N}_{3} \mathrm{O}_{5}: \mathrm{C}$, 58.36; H, 4.59; N, 12.76\%. Found: C, 58.50; H, 4.71; N, 12.53\%. ${ }^{1} \mathrm{H}$ NMR $\left(500.1 \mathrm{MH}_{\mathrm{Z}}\right.$, $\left.\mathrm{CDCl}_{3}\right): \delta=1.06\left(3 \mathrm{H}, \mathrm{t},{ }^{3} J_{\mathrm{HH}}=7 \mathrm{H}_{\mathrm{Z}}, \mathrm{CH}_{3}\right), 2.31\left(3 \mathrm{H}, \mathrm{s}, \mathrm{CH}_{3}\right), 3.98\left(2 \mathrm{H}, \mathrm{q},{ }^{3} J_{\mathrm{HH}}=7 \mathrm{H}_{\mathrm{Z}}\right.$, $\left.\mathrm{CH}_{2}\right), 4.13\left(\mathrm{H}, \mathrm{d},{ }^{3} J_{\mathrm{HH}}=7.1 \mathrm{H}_{\mathrm{Z}}, \mathrm{CH}\right), 4.18\left(\mathrm{H}, \mathrm{d},{ }^{3} \mathrm{~J}_{\mathrm{HH}}=7.1 \mathrm{H}_{\mathrm{Z}}, \mathrm{CH}\right), 7.31-8.06(4 \mathrm{H}, \mathrm{m}$, aromatic), $10.28\left(1 \mathrm{H}\right.$, broad s, NH). ${ }^{13} \mathrm{C} \mathrm{NMR}\left(125.7 \mathrm{MH}_{\mathrm{Z}}, \mathrm{CDCl}_{3}\right): \delta=14.48$ and 19.00 $\left(2 \mathrm{CH}_{3}\right), 41.01$ and $41.71(2 \mathrm{CH}), 61.01\left(\mathrm{CH}_{2}\right), 105.54(\mathrm{CN}), 114.59$ and $144.77(\mathrm{C}=\mathrm{C})$, $124.42,129.40,148.12,149.04$ (aromatic), 162.34 and $165.70(2 \mathrm{C}=\mathrm{O})$.

Ethyl 5-cyano-2-methyl-6-oxo-4-(4-nitrophenyl)-1,6-dihydropyridine 3-carboxylate (7B)

White powder, $(37 \%)$, m.p. $198-200^{\circ} \mathrm{C}$, IR $(\mathrm{KBr})\left(v_{\max } / \mathrm{cm}^{-1}\right): 3395(\mathrm{NH}), 2225(\mathrm{CN}), 1721$ $(\mathrm{C}=\mathrm{O}), 1348$ and $1593\left(\mathrm{NO}_{2}\right)$. MS, m/z $(\%): 327\left(\mathrm{M}^{+}, 3\right)$. Anal. Calcd for $\mathrm{C}_{16} \mathrm{H}_{13} \mathrm{~N}_{3} \mathrm{O}_{5}: \mathrm{C}$, 58.72; H, 4.00; N, 12.84\%. Found: C, 58.55; H, 4.15; N, 12.99\%. ${ }^{1} \mathrm{H}$ NMR $\left(500.1 \mathrm{MH}_{\mathrm{Z}}\right.$, $\left.\mathrm{CDCl}_{3}\right): \delta=0.88\left(3 \mathrm{H}, \mathrm{t},{ }^{3} J_{\mathrm{HH}}=7 \mathrm{H}_{\mathrm{Z}}, \mathrm{CH}_{3}\right), 2.66\left(3 \mathrm{H}, \mathrm{s}, \mathrm{CH}_{3}\right), 3.96\left(2 \mathrm{H}, \mathrm{q},{ }^{3} J_{\mathrm{HH}}=7 \mathrm{H}_{\mathrm{Z}}\right.$, $\left.\mathrm{CH}_{2}\right), 7.25-8.37\left(4 \mathrm{H}, \mathrm{m}\right.$, aromatic), $13.54\left(1 \mathrm{H}\right.$, broad s, NH). ${ }^{13} \mathrm{C} \mathrm{NMR}\left(125.7 \mathrm{MH}_{\mathrm{Z}}\right.$, $\left.\mathrm{CDCl}_{3}\right): \delta=13.96$ and $18.87\left(2 \mathrm{CH}_{3}\right), 62.46\left(\mathrm{CH}_{2}\right), 105.12(\mathrm{CN}), 114.18,124.40,129.01$, $142.17,149.04,154.22,159.33,162.47$ (aromatic and olefinic), 164.57 and $165.48(2 \mathrm{C}=\mathrm{O})$.

\section{Results and Discussion}

Herein we report a new and efficient one-pot synthesis of polysubstituted dihydropyridones derivatives by four-component reaction between cyanoacetamide, aryl aldehydes and ethyl acetoacetate with ammonium acetate using nano $\mathrm{ZnO}$. The reaction was performed in ethanol under reflux conditions and afforded good yields of products. (Figure 1).

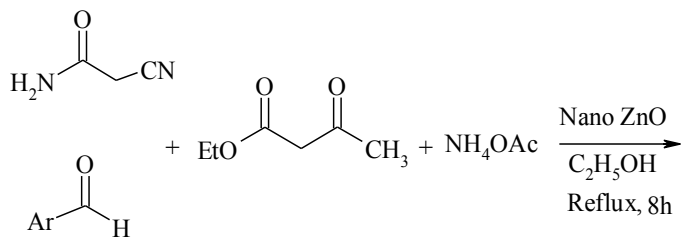<smiles>CCOC(=O)C1=C(C)NC(=O)C(C#N)C1(C)C#N</smiles>

(A)<smiles>CCOC(=O)c1c(C)[nH]c(=O)c(C#N)c1[N]</smiles>

(B)

\begin{tabular}{ccc}
\hline Entry & $\mathrm{Ar}$ & 8 yield (A/B) \\
\hline 1 & $\mathrm{C}_{6} \mathrm{H}_{5}$ & $59($ Only A) \\
2 & $4-\mathrm{Cl} \mathrm{C}_{6} \mathrm{H}_{4}$ & 58 (Only A) \\
3 & $4-\mathrm{Br} \mathrm{C}_{6} \mathrm{H}_{4}$ & 61 (Only A) \\
4 & $3-\mathrm{Meo}_{6} \mathrm{H}_{4}$ & 57 (Only A) \\
5 & $2-\mathrm{NO}_{2} \mathrm{C}_{6} \mathrm{H}_{4}$ & 54 (Only A) \\
6 & $3-\mathrm{NO}_{2} \mathrm{C}_{6} \mathrm{H}_{4}$ & 68 (Only A) \\
7 & $4-\mathrm{NO}_{2} \mathrm{C}_{6} \mathrm{H}_{4}$ & $63 / 37$ \\
\hline \multicolumn{3}{c}{$*$ Isolated yield }
\end{tabular}

Figure 1 Four-component reaction between cyanoacetamide, aryl aldehydes and ethyl acetoacetate with ammonium acetate using nano $\mathrm{ZnO}$.

The reaction course without ammonium acetate in the absence of zinc oxide a complex mixture was obtained from which no product was isolated. The reaction course in the presence of ammonium acetate without zinc oxide afforded the product in lower yield and longer reaction time. Using nano $\mathrm{ZnO}$ afforded the product in higher yield and shorter 
reaction time. The structure of compounds $\mathbf{A}$ and $\mathbf{B}$ was deduced from their elemental analyses and their IR, ${ }^{1} \mathrm{H}$, and ${ }^{13} \mathrm{CNMR}$ spectra data. The mass spectrum of compound $7 \mathbf{A}$ displayed the molecular ion peak at $m / z=329$ as the base peak. The $500 \mathrm{MH}_{\mathrm{Z}}{ }^{1} \mathrm{H}$ NMR spectrum of compound 7A exhibited a $\mathrm{D}_{2} \mathrm{O}$-exchangable broad signal at $10.28 \mathrm{ppm}$ for $\mathrm{NH}$ proton, and displays one sharp line $(\delta 2.31 \mathrm{ppm})$ for the methyl group. Ethyl protons were observed as a triplet $\left({ }^{3} J_{H H}=7 \mathrm{H}_{\mathrm{Z}}\right)$ at $1.06 \mathrm{ppm}$ and a quartet at $3.98 \mathrm{ppm}$. Two doublet were observed respectively at 4.13 and $4.18 \mathrm{ppm}$ for methine protons. $\left({ }^{3} J_{H H}=7.1 \mathrm{H}_{\mathrm{Z}}\right)$. Aromatic protons resonated between 7.31 and $8.06 \mathrm{ppm}$ as multiplets. The ${ }^{13} \mathrm{C}$ NMR spectrum of compound 7A showed 14 distinct resonances in agreement with the proposed structure. The IR spectrum showed an absorption bond at $3407 \mathrm{~cm}^{-1}$ for NH group. The carbonyl stretching vibrations observed as strong absorption bonds at $1715 \mathrm{~cm}^{-1}$. The nitrile stretching vibrations observed absorption bond at $2255 \mathrm{~cm}^{-1}$. The nitro stretching vibrations observed absorption bonds at 1345 and $1515 \mathrm{~cm}^{-1}$. The mass spectrum of compound $7 \mathbf{B}$ displayed the molecular ion peak at $m / z=327$ as the base peak. The $500 \mathrm{MH}_{\mathrm{Z}}{ }^{1} \mathrm{H}$ NMR spectrum of compound $7 \mathbf{B}$ exhibited a $\mathrm{D}_{2} \mathrm{O}$-exchangable broad signal at $13.54 \mathrm{ppm}$ for $\mathrm{NH}$ proton, and displays one sharp line $(\delta 2.66 \mathrm{ppm})$ for the methyl group. Ethyl protons were observed as a triplet $\left({ }^{3} J_{H H}\right.$ $=7 \mathrm{H}_{\mathrm{Z}}$ ) at $0.88 \mathrm{ppm}$ and a quartet at $3.96 \mathrm{ppm}$. Aromatic protons resonated between 7.25 and $8.37 \mathrm{ppm}$ as multiplets. The ${ }^{13} \mathrm{C}$ NMR spectrum of compound $7 \mathbf{B}$ showed 14 distinct resonances in agreement with the proposed structure. The IR spectrum showed an absorption bond at $3395 \mathrm{~cm}^{-1}$ for $\mathrm{NH}$ group. The carbonyl stretching vibrations observed as strong absorption bonds at $1721 \mathrm{~cm}^{-1}$. The nitrile stretching vibrations observed absorption bond at $2225 \mathrm{~cm}^{-1}$. The nitro stretching vibrations observed absorption bonds at 1348 and $1593 \mathrm{~cm}^{-1}$. Although the mechanistic details of the above reaction are not known, a plausible mechanism may be advanced to rationalize product formation.

Resumably a intermediate 3 formed from michael addition of product $\mathbf{1}$ the addition of cyanoacetamide with aryl aldehydes using nano $\mathrm{ZnO}$ and product $\mathbf{2}$ the addition of ethyl acetoacetate with ammonium acetate which could undergo stepwise cyclization to produce $\mathbf{A}$ by elimination of $\mathrm{NH}_{3}$. The amide tautomer is considerably more stable. Admittedly the exchangeable peak in the 1H NMR spectrum is very high for a $\mathrm{NH}$ peak, and would appear to be more consistent with a $\mathrm{OH}$ peak. The A product is finally converted to $\mathbf{B}$ by oxidation (Figure 2).
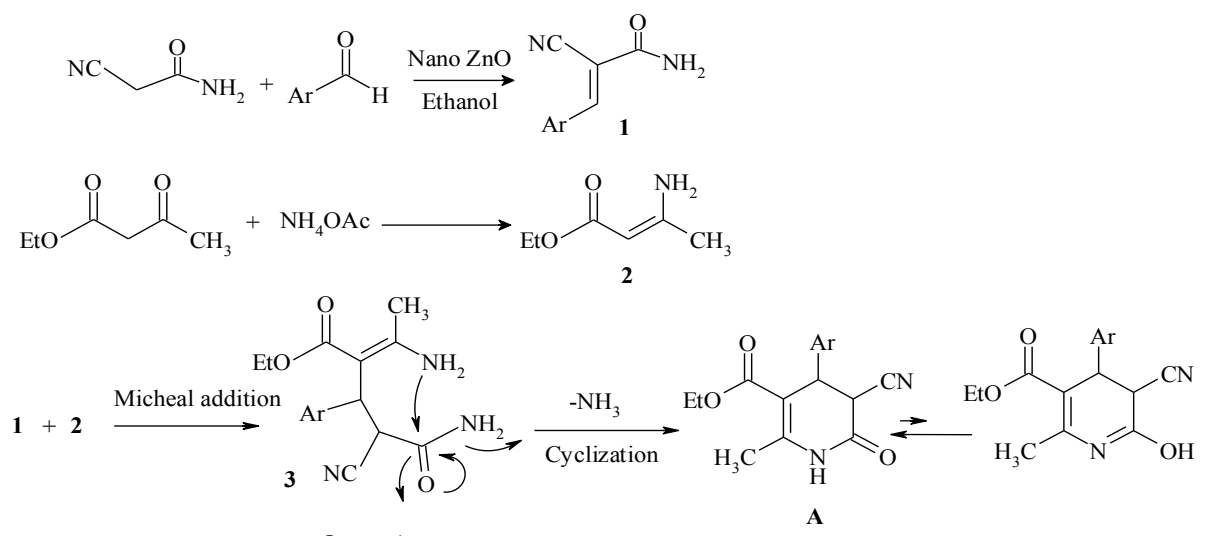<smiles>Cc1[nH]c(=O)c(C(=O)O)c(C)c1C(=O)O</smiles>

Figure 2. Suggested mechanism for formation of compound $\mathbf{A}$ and $\mathbf{B}$. 


\section{Conclusion}

In summary, here we reported a four-component reaction between cyanoacetamide, aryl aldehydes and ethyl acetoacetate with ammonium acetate using nano $\mathrm{ZnO}$. The reaction was performed in ethanol under reflux conditions and afforded good yields of products. The present method carries the advantage that not only is the reaction performed under neutral conditions but also that the substances can be mixed without any activation or modification. nano $\mathrm{ZnO}$ was prepared as previously described in the literature. ${ }^{16}$

\section{Acknowledgment}

We gratefully acknowledge financial support from the Research Council of Islamic Azad University, Yazd branch and the Islamic Azad University, Zahedan branch.

\section{References}

1. Misic-Vukovic M, Mijin D, Radojkovic-Velickovic M, Valentic N and Krstic V, $J$ Serb Chem Soc., 1998, 63, 585.

2. Balasubramanian M, Keay J G, Katritzky A R, Rees C W and Scriven E V F, Comprehensive Heterocyclic Chemistry II Vol. 5, Pergamon Press, London, 1996, 245-300 Chapter 6.

3. Constable E C, Housecroft C E, Neuburger M, Phillips D, Raithby P R, Schofield E, Sparr E, Tocher D A, Zehnder M and Zimmermann Y, J Chem Soc Dalton Trans., 2000, 13, 2219.

4. Kim B Y; Ahn J B, Lee H W, Kang S K, Lee J H, Shin J S, Ahn S K, Hong C I and Yoon S.S, Eur J Med Chem., 2004, 39, 433.

5. Enyedy I J, Sakamuri S, Zaman W A, Johnson K M and Wang S, Bioorg Med Chem Lett., 2003, 13, 513.

6. Pillai A D, Rathod P D, Franklin P X, Patel M, Nivsarkar M, Vasu K K, Padh H and Sudarsanam V, Biochem Biophys Res Commun., 2003, 301, 183.

7. Klimešová V, Svoboda M, Waisser K, Pour M and Kaustová J, Fármaco., 1999, 54, 666.

8. Jones G, Katritzky A R, Rees C W and Scriven E V F, Editors, Comprehensive Heterocyclic Chemistry II Vol. 5, Pergamon Press, London, 1996), 168-243 Chapter 5 and references cited therein.

9. Katritzky A R, Abdel-Fattah A A A, Tymoshenko D O and Essawy S A, Synth., 1999, $\mathbf{1 2}, 2114$.

10. Rong L, Han H X, Jiang H, Zhang Q and Tu S, Synth Común., 2009, 39, 1027.

11. Look D C, Reynolds D C, Sizelove J R, Jones R L, Litton C W, Cantwell C, et al, Solid State Común., 1998, 105, 399.

12. Gouvea C A K, Wypych F, Moraes S G, Duran N, Nagata N and Peralta Z P, Chemosphere, 2000, 40, 433.

13. Huang M H, Mao S, Feick H, Yan H, Wu Y, Kind H, et al, Room-temperature ultraviolet nanowire nanolasers. Science, 2001, 292, 1897.

14. Rensmo H, Keis K, Lindstrom H, Sodergren S, Solbrand A, Hagfeldt A, et al, J Phys Chem B, 1997, 101, 2598.

15. Kind H, Yan H; Messer B; Law M and Yang P, Adv Mater., 2002, 14, 158.

16. Tang, E.; Tian, B.; Zheng, E.; Fu, C.; and Cheng, G., Chem. Eng. Comm., 2008, 195, 479. 


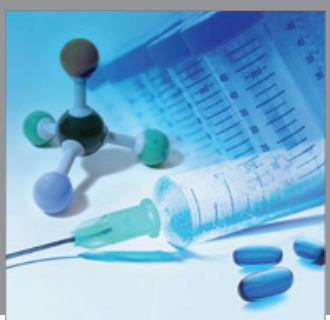

International Journal of

Medicinal Chemistry

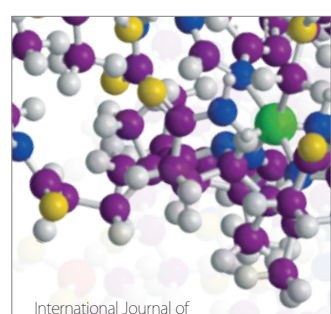

Carbohydrate Chemistry

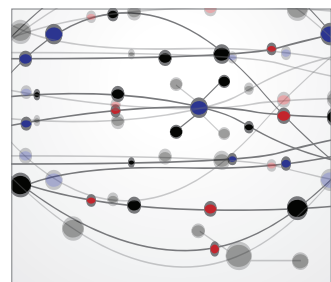

The Scientific World Journal
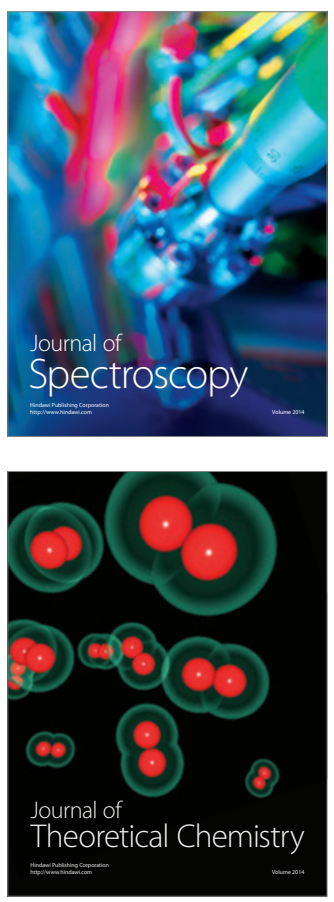
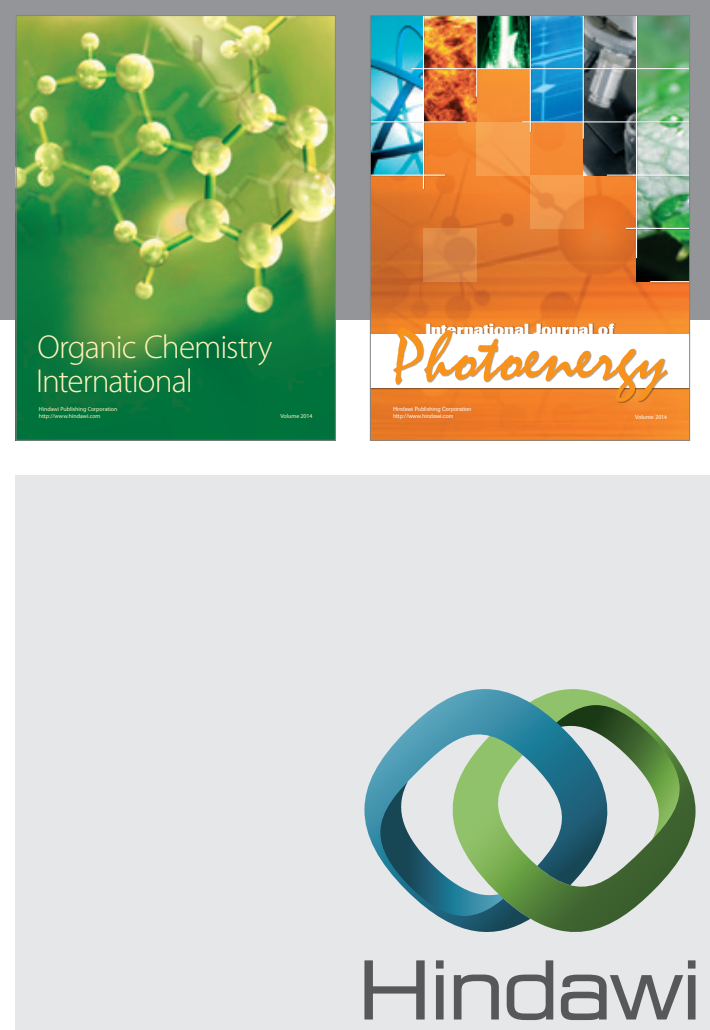

Submit your manuscripts at

http://www.hindawi.com
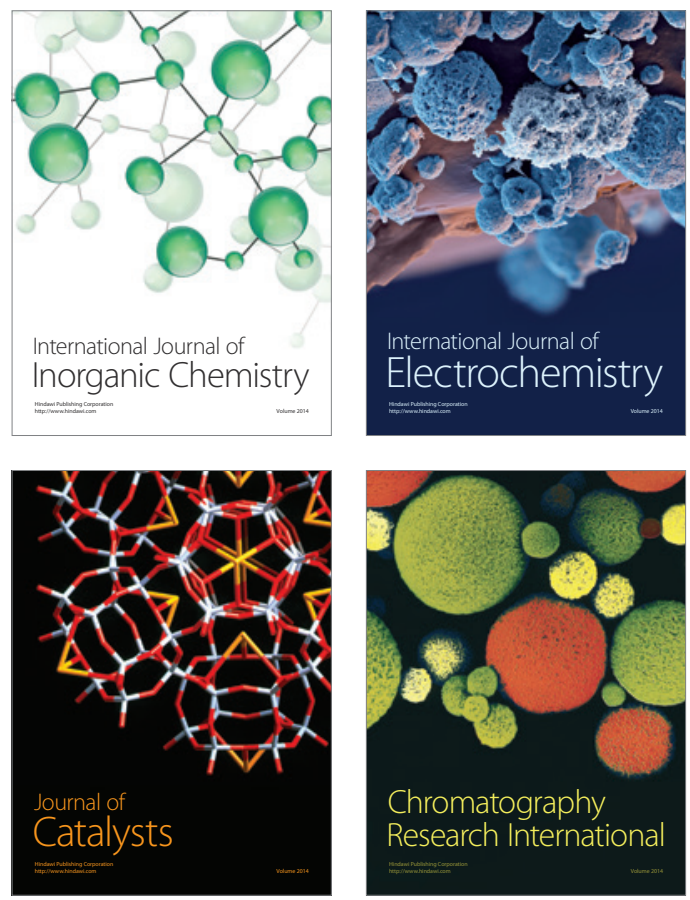
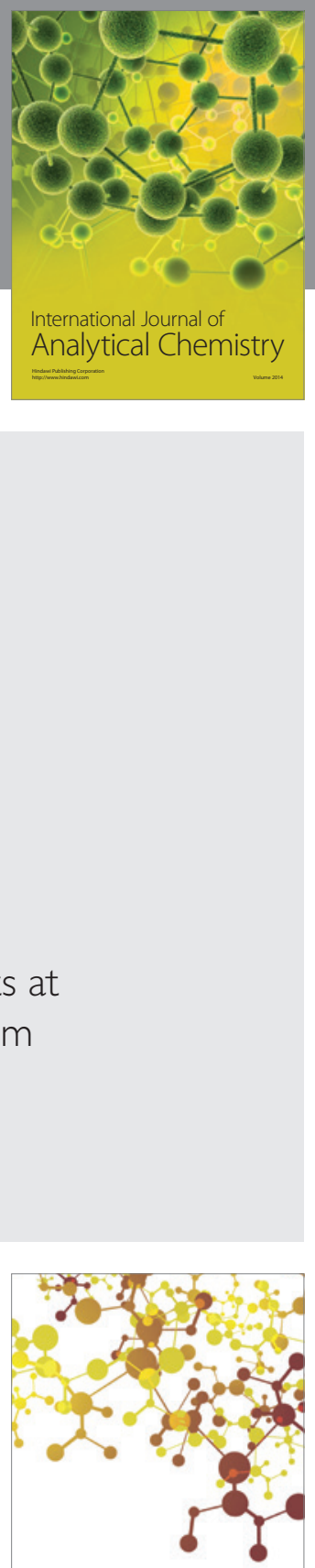

Journal of

Applied Chemistry
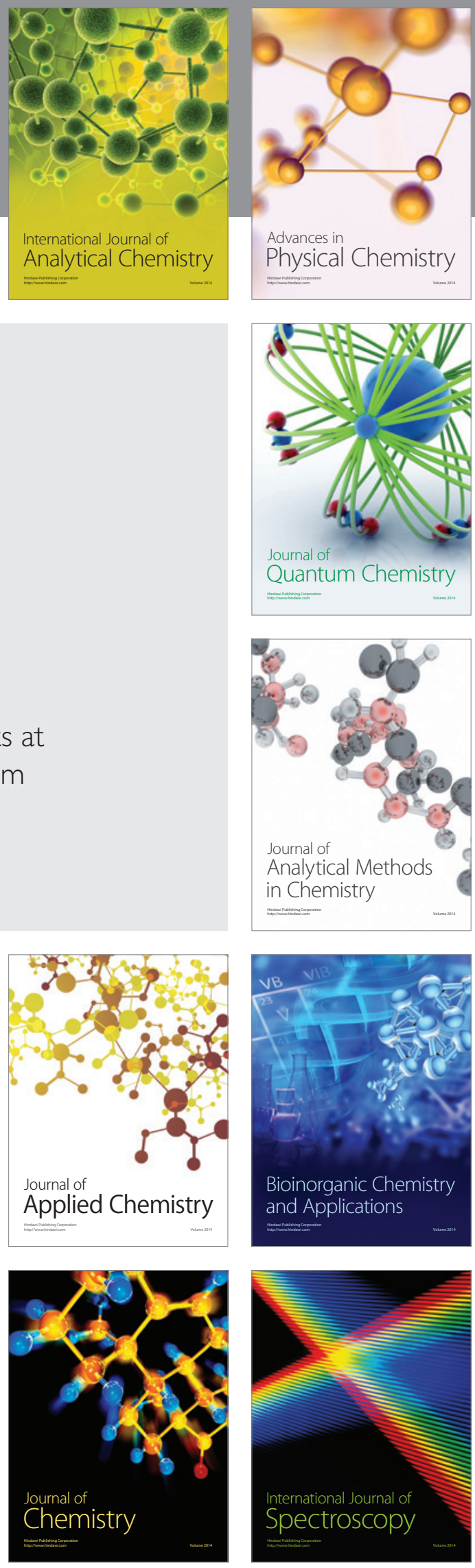